\title{
RADIO MEASUREMENTS OF POSSIBLE PROTO-PLANETARY NEBULAE
}

\author{
C.R. Purton and P.A. Feldman \\ York University, Toronto, Canada, and Herzberg Institute of \\ Astrophysics, NRC, Ottawa, Canada, respectively.
}

A number of objects which may be proto-planetary nebulae are being studied. They were found through a radio search of early-type emissionline stars, and the radio emission is believed to originate in extensive circumstellar ionised gas. The objects have an infrared spectrum which indicates the presence of dust, and a continuum radio spectrum characteristic of prolonged mass outflow from the central star. Of the five objects discussed here, Vy2-2, Hb12, HD167362, H1-36 and V1016 Cygni, a distance is available for only the last: for that object the calculated mass of the ionized gas is .02 $M_{\odot}$ (see Ahern et al, 1977). If this is typical of the five, then they may represent the early stages of the low-mass planetary nebulae discussed by Wood and Cahn (1977). References: (1) Ahern, F.A., Fitzgerald, P.M., Marsh, K.A., and Purton, C.R. 1977, Astron. \& Astrophys., in press. (2) Wood, P.R., and Cahn, J.H. 1977, Astrophys. J. 211, 499 .

\section{DISCUSSION}

Kwok: I would like to comment on Dr. Zuckerman's criteria for protoplanetary nebulae. He suggested that the formation of a planetary nebula is the result of continuous mass loss of $10^{-5} \mathrm{M}_{\odot} \mathrm{yr}^{-1}$ over a period of $10^{5}$ years. If we adopt an ejection velocity of $10 \mathrm{kms}^{-1}$, the size of the circumstellar envelope is greater than 1 pc. This can certainly be responsible for the $\mathrm{CO}$ and dust emission in the outer parts of NGC 7027, but unlikely to be responsible for the nebula itself, which is more likely to be the result of a massive ejection within a short period of time. In conclusion, a red giant with a mass loss rate of

e.g. $10^{-6} \mathrm{M}_{\odot} \mathrm{yr}^{-1}$ should not be excluded from the possibility of becoming a planetary nebula and planetary nebulae will not be formed exclusively from carbon stars.

Purton: The mass loss rate determined for V1016 Cygni is $3 \times 10^{-5} \mathrm{M}_{\odot} \mathrm{yr}^{-1}$, but apparently this mass loss continued only a few hundred years.

Feldman: I would like to emphasize the opportunity we now have in the case of HM Sge to study the early stages of formation of a possible proto-planetary object like V1016 Cyg. HM Sge brightened by $\geq 5 \mathrm{~m}$ during 1975 and has an emission line spectrum quite similar to that of a highdensity planetary nebula. Recently, in May 1977, I have found a continuum radio source associated with this object. The radio source, of strength $\sim 40 \mathrm{mJy}$ at $10.5 \mathrm{GHz}$, apparently was not present in November 1977 . Thus the radio emission has emerged several years after the optical outburst. 